\title{
Predicting Lettuce Canopy Photosynthesis with Statistical and Neural Network Models
}

\author{
Jay Frick, ${ }^{1}$ Cyrille Precetti, ${ }^{2}$ and Cary A. Mitchell ${ }^{3}$ \\ National Aeronauticsand Space Administration Specialized Center of Researchand Training in Bioregenerative \\ Life Support, Purdue University, 1165 Horticulture Building, West Lafayette, IN 47907-1165
}

\begin{abstract}
Adortional, INDEX woRDs. Lactuca sativa, CELSS, chaos, crop optimization, environmental control, hydroponics, NASA
Abstract. An artificial neural network (NN) and a statistical regression model were developed to predict canopy photosynthetic rates (Pn) for 'Waldman's Green' leaf' lettuce (Latuca sativa L.). All data used to develop and test the models were collected for crop stands grown hydroponically and under controlled-environment conditions. In the NN and regression models, canopy $\mathrm{Pn}$ was predicted as a function of three independent variables: shootzone $\mathrm{CO}_{2}$ concentration (600 to $\left.1500 \mathrm{mmol} \cdot \mathrm{mol}^{-1}\right)$, photosynthetic photon flux (PPF) $\left(600\right.$ to $\left.1100 \mu \mathrm{mol} \cdot \mathrm{m}^{-2} \cdot \mathrm{s}^{-1}\right)$, and canopy age $(10$ to 20 days after planting). The models were used to determine the combinations of $\mathrm{CO}_{2}$ and PPF setpoints required each day to maintain maximum canopy Pn. The statistical model (a third-order polynomial) predicted Pn more accurately than the simple NN (a three-layer, fully connected net). Over an 11-day validation period, average percent difference between predicted and actual Pn was $12.3 \%$ and $24.6 \%$ for the statistical and NN models, respectively. Both models lost considerable accuracy when used to determine relatively long-range Pn predictions ( $\geq 6$ days into the future).
\end{abstract}

The U.S. National Aeronautics and Space Administration (NASA) is considering the use of higher plants to sustain human life during long-term space exploration and colonization missions (Salisbury and Clark, 1996). The proposed controlled environment life-support system (CELSS) will be at least partially bioregenerative and feasible during missions when resupply from Earth is either impossible or impractical. Through the biological processes of photosynthesis and transpiration, higher plants in a CELSS will absorb $\mathrm{CO}_{2}$, release $\mathrm{O}_{2}$, produce fresh food, and provide a continual source of potable water (Hoff et al., 1983).

Space structures typically are designed with major emphasis on weight reduction (Allen and Haisler, 1985). The resulting constraints suggest that a CELSS should be as small as possible, with minimal resource storage buffers. This further implies a highly dynamic system, capable of responding rapidly to changes in state or demand. To maintain stability and manage such a biomechanical system, comprehensive crop-response models are needed. Such models must accurately predict real-time crop responses with respect tochanges in the environment and the changing needs of attending space travelers.

Because plants are living organisms, predictable and consistent crop responses often are difficult to obtain. It is well known that crop dynamics are a function of genetic diversity as well as complex interactions with the environment (Milthorpe and Moorby, 1979). However, other chaotic factors also could play a role. For example, it is unknown if subtle environmental variations early in the growth cycle influence long-term crop responses. All such factors should be addressed when developing comprehensive crop-response models for CELSS design and simulation.

In this study, we developed a simple neural network (NN) as an alternative to a statistical regression model to describe photosynthetic response (Pn) during crop development. NNs form a unique class of

Received for publication 20ct. 1997. Accepted for publication 18 May 1998. Purdue University Agricultural Experiment Station journal paper no. 15489. Research supported in part by NASA grant NAGW-2329. The cost of publishing this paper was defrayed in part by the payment of page charges. Under postal regulations, this paper therefore must be hereby marked advertisement solely to indicate this fact.

'Research engineer. Current address: Dept. of Biological and Agricultural Engineering, North Carolina State University, Raleigh, NC 27695.

${ }^{2}$ Postdoctoral research assistant, Dept. of Agricultural Engineering, Purdue University.

Professor, Dept. of Horticulture, Purdue University; to whom reprint requests should be addressed. mathematical algorithms constructed from sets of simple computing elements thathave been assembled to perform a specific task (Machado et al., 1992). Information is processed as it passes through each of the elements (or neurons) within the network. NNs were developed by McCulloch and Pitts (1943), who described the computing elements as analogous to biological neurons in the human brain.

The motivation for using NNs lies in their learning ability. A mathematical proof exists that demonstrates that any continuous function can be reproduced (or approximated) with a three-layer $\mathrm{NN}$, provided enough neurons are in the hidden layer. Recently, NNs have been reported to learn input-output relationships without the statistics required for a regression approach (Bailey and Thompson, 1990a). NN models can be developed when there is limited information about the relationship between independent and dependent variables (Sarle, 1994), and they can be retrained as additional experimental data become available. In contrast, incorporating new data into an existing statistical model is difficult, unless entire series of experiments are carefully preplanned for that purpose. A drawback of NNs is that they provide no information regarding statistical significance of independent variables or significance of interactions among the variables, nor do they offer any insight into the biological or physical processes underlying a given input-output data set. Additional information detailing the relationship between statistics and NNs can be found in Ripley (1993), Weiss and Kulikowski (1991), and White (1992).

The objective of this study was to develop and evaluate dynamic models to quantify lettuce canopy Pn. Models incorporating multiple interdependent variables and those capable of near real-time $\mathrm{Pn}$ predictions were of primary interest. Algorithms that predict crop response and yield are needed for CELSS development and are relevant in other applications of controlled-environment agriculture as well. For example, these efforts may serve as a precursor to phasic environmental control strategies or for active-control scenarios, whereby environmental conditions are optimized in real time, depending on continuous feedback response signals from the crop.

\section{Materials and Methods}

EquiPMENT AND APPARATUS. Lettuce canopy net Pn data were generated from experiments conducted using the controlled-environment Minitron II facility at Purdue University (Knight et al., 
1988). The Minitron II system includes two independent and precisely controlled transparent plant-growth chambers retrofitted for automatic computer control and data acquisition capabilities.

Each cylindrical Minitron chamber uses recirculating bulk-liquid hydroponic culture and a growth surface area of $0.147 \mathrm{~m}^{2}$. The Minitrons and support equipment provide control over the following environmental variables: irradiance, shootzone temperature, shootzone $\mathrm{CO}_{2}$ concentration, atmospheric gas turnover rate, and nutrient solution $\mathrm{pH}$. Nutrient solution temperatures are monitored but not controlled. The Minitrons have sealable compartments that separate the rootzone from the shootzone, allowing gas-exchange measurements in either compartment without cross contamination. Additional information and specifications of the Minitrons and their associated support equipment have been reported previously (Knight et al., 1988).

Automatic data acquisition and process control was achieved with Paragon software for industrial automation (Intec Controls Corp., Walpole, Mass.). The software was run on a Northgate $386 \mathrm{PC}$ (Northgate Computer Systems, Minneapolis, Minn.). Incoming data and control signals were conditioned and interfaced to the computer through Opto-22 I/O modules and brain boards (Opto 22, Temecula, Calif.).

Values for canopy Pn were automatically calculated and averaged over 10 -min sampling intervals, enabling a near real-time evaluation of changes in Pn without mechanically disturbing the canopy. Gasexchange measurements were corrected for standard temperature and pressure. The procedure and generic equation (Eq. [1]) used to determine steady-state canopy Pn in the Minitrons has been described previously (Knight et al., 1988).

$\mathrm{Pn}=\mathrm{CO}_{2}$ absorbed $\times$ air flow rate/growing area

Correcting $\mathrm{CO}_{2}$ values for standard temperature and pressure, assuming ideal-gas approximations $(1 \mathrm{~mol} / 22.4 \mathrm{~L})$, and converting units resulted in $\mathrm{Pn}$ in terms of $\mu \mathrm{mol} \cdot \mathrm{m}^{-2} \cdot \mathrm{s}^{-1} \mathrm{CO}_{2}$, where area $\left(\mathrm{m}^{2}\right)$ refers to the total growing surface area in a Minitron chamber.

EXPERIMENTAL PROCEDURE. Lettuce seeds ('Waldman's Green') were sown directly into closed-cell Ethafoam plugs floated in quarter-strength Hoagland's no. I nutrient solution with a pH of 5.5 (Hoagland and Arnon, 1950). Seeds were imbibed with this nutrient solution, which was transferred by capillary action through a narrow strip of polyester wicking material in each plug. A single layer of plastic wrap was placed loosely over the reservoir surface to ensure high humidity for the germinating seedlings. The reservoir and seeded plugs were placed under a 60 -W incandescent bulb (25 $\mu \mathrm{mol} \cdot \mathrm{m}^{-2} \cdot \mathrm{s}^{-1}$ ) for a 24 -h germination period.

After $24 \mathrm{~h}, 12$ seeded plugs were transferred to holders in the hydroponic container $\left(0.147 \mathrm{~m}^{2}\right)$ of each Minitron. Chambers were then sealed with a transparent lid and then irradiated overhead for the remainder of the experiment. Nutrient solution reservoirs were filled with half-strength Hoagland's nutrient solution $(\mathrm{pH} 6.0$ and $\mathrm{EC}=$ $1300 \mathrm{mS} \cdot \mathrm{cm}^{-1}$ ), which was continuously aerated with ambient air at $1.5 \mathrm{~L} \cdot \mathrm{min}^{-1}$. Nutrient solution was recirculated to a sealed external reservoir where $\mathrm{pH}$ was automatically maintained between 5.8 and 6.3 .

On days 1 to 5, photosynthetic photon flux (PPF) was set at 250 $\mu \mathrm{mol} \cdot \mathrm{m}^{-2} \cdot \mathrm{s}^{-1}$ and was provided by a combination of $400-\mathrm{W}$ metal halide and 150-W incandescent flood lamps for $20 \mathrm{~h} \cdot \mathrm{d}^{-1}$. Day shootzone temperature was $26^{\circ} \mathrm{C}$ and night temperature was $23^{\circ} \mathrm{C}$. Rootzone temperature was not controlled but was typically $1{ }^{\circ} \mathrm{C}$ higher than that of the shootzone. Shootzone $\mathrm{CO}_{2}$ was maintained at $600 \mu \mathrm{mol} \cdot \mathrm{mol}^{-1}$, and atmospheric turnover rate of shootzone air was held constant at $8.5 \mathrm{~L} \cdot \mathrm{min}^{-1}$.

On day 6 , solution reservoirs were drained and immediately refilled with single-strength Hoagland's nutrient solution, $\mathrm{EC}=2500$ $\mathrm{mS} \cdot \mathrm{cm}^{-1}$ (Hoagland and Arnon, 1950) and PPF was increased to 600 $\mu \mathrm{mol} \cdot \mathrm{m}^{-2} \cdot \mathrm{s}^{-1}$. PPF was provided by a combination of high pressure sodium, metal halide, and incandescent flood lamps. The incandescent output was continuously variable, making fine gradations in PPF possible. All other environmental conditions were controlled to the same setpoints as described. Environmental conditions in both Minitron chambers were maintained at the above levels until day 10 after planting.

Treatments to enhance photosynthesis began on day 10 after planting. Day 10 was chosen because canopy Pn was not detectable before this time due to relatively small plant size compared to the total chamber volume. Treatments consisted of altering PPF and $\mathrm{CO}_{2}$ levels, and then recording the corresponding steady-state canopy $\mathrm{Pn}$ response associated with each treatment combination. Three PPF levels were investigated: 600,850 , and $1100 \mu \mathrm{mol} \cdot \mathrm{m}^{-2} \cdot \mathrm{s}^{-1}$. The $\mathrm{CO}_{2}$ concentrations examined were 600,1050 , and $1500 \mu \mathrm{mol} \cdot \mathrm{mol}^{+}{ }^{2}$. Each day the full factorial of $\mathrm{CO}_{2}$ and PPF (nine treatment combinations) was run within each Mintron chamber. Each treatment combination was maintained for 50 consecutive minutes after steady-state conditions were achieved. The resulting canopy Pn was measured repeatedly and averaged over these 50 -min treatment intervals.

The order of $\mathrm{PPF}$ and $\mathrm{CO}_{2}$ treatment combinations was randomly selected at the beginning of each day. After Pn data had been collected for all nine treatment combinations, setpoints for PPF and $\mathrm{CO}_{2}$ were reset to $850 \mu \mathrm{mol} \cdot \mathrm{m}^{-2} \cdot \mathrm{s}^{-1}$ and $1050 \mu \mathrm{mol} \cdot \mathrm{mol}^{-1}$, respectively, for the remainder of the day's 20 -h photoperiod. This entire procedure was repeated each day, starting on day 10 and ending on day 20 . The Pn data derived from this experiment were used to develop the regression and NN models.

In a subsequent experiment, the procedure was duplicated in as exact a manner as possible, except that daily treatments consisted of setting $\mathrm{CO}_{2}$ and PPF setpoints to levels predicted by the models to result in maximum canopy $P n$. Each day from day 10 to 20 , the maxPn treatment setpoints (via both models) were run within each Minitron chamber, and corresponding canopy Pn was measured. The data from this second experiment were used to evaluate the performance and accuracy of both the statistical and NN models.

The accuracy of each model was evaluated in two ways: First, the absolute difference between predicted and actual Pn (e.g., predicted maximum $\mathrm{Pn}$ - measured $\mathrm{Pn}$ ) was determined for each day during the evaluation period (days 10 to 20 after planting). Therefore, Pn differences that approached 0 indicated a highly accurate prediction by the model. Secondly, percent differences (Holman, 1984) between predicted and actual Pn were calculated for each model, and an overall average percent difference was calculated over the $10-\mathrm{d}$ test period.

Model development. The statistical, regression model was developed using SAS software (SAS Institute, Cary, N.C.). Experimental design was treated as a split block, with each Minitron chamber as a separate experimental unit. Main effects and interactions among the dependent variables were completely partitioned into 198 degrees of freedom (Steel and Torrie, 1980). Next, a Bartlet's test was used to check for error homogeneity (Steel and Torrie, 1980). Since error variance increased with the mean, the data were transformed (using a square-root function) in accordance with the Box-Cox analysis of transformations (Box et al., 1978). After generating the ANOVA tables, insignificant terms $(P=0.05)$ were eliminated. In the final step, best-fit regression coefficients were calculated to complete the Pn model.

Developing the $\mathrm{NN}$ model was largely a trial-and-error process. From early simulations we determined that relatively simple NN topology would suffice to represent our Pn data. The raw data were 
Table 1. Regression model ${ }^{7}$ used to predict lettuce canopy photosynthesis (Pn). Pn equation is valid only when independent variables are within given limits.

\begin{tabular}{lll}
\hline \hline Term no. & Model term & Coefficient \\
\hline 1 & Intercept $=31.135$ & --- \\
2 & Time & -5.335058 \\
3 & Time $^{2}$ & 0.352644 \\
4 & Time $^{3}$ & $-8.526 \times \mathrm{E}-03$ \\
5 & $\mathrm{CO}_{2}$ & $-2.7849 \times \mathrm{E}-02$ \\
6 & $\mathrm{CO}_{2} \times \mathrm{time}^{2}$ & $4.388 \times \mathrm{E}-03$ \\
7 & $\mathrm{CO}_{2} \times \mathrm{time}^{2}$ & $-2.61 \times \mathrm{E}-04$ \\
8 & $\mathrm{CO}_{2} \times \mathrm{time}^{3}$ & $6.118 \times \mathrm{E}-06$ \\
9 & $\mathrm{PPF}^{2}$ & $-6.5942 \times \mathrm{E}-02$ \\
10 & $\mathrm{PPF}^{2}$ & $1.988 \times \mathrm{E}-05$ \\
11 & $\mathrm{PPF}^{2} \times \mathrm{time}^{2}$ & $8.532 \times \mathrm{E}-03$ \\
12 & $\mathrm{PPF}^{2} \times \mathrm{time}^{2}$ & $-3.95 \times \mathrm{E}-04$ \\
13 & $\mathrm{PPF}^{2} \mathrm{time}^{3}$ & $8.799 \times \mathrm{E}-06$ \\
14 & $\mathrm{PPF}^{2} \times \mathrm{time}^{2}$ & $-1.386 \times \mathrm{E}-06$ \\
15 & $\mathrm{CO}_{2} \times \mathrm{PPF}^{2}$ & $7.9839 \times \mathrm{E}-05$ \\
16 & $\mathrm{CO}_{2} \times \mathrm{PPF}^{2}$ & $-1.6702 \times \mathrm{E}-08$ \\
17 & $\mathrm{CO}_{2} \times \mathrm{PPF}^{2} \times \mathrm{time}_{2}$ & $-1.2236 \times \mathrm{E}-05$ \\
18 & $\mathrm{CO}_{2} \times \mathrm{PPF}_{1} \times \mathrm{time}^{2}$ & $6.96 \times \mathrm{E}-07$ \\
19 & $\mathrm{CO}_{2} \times \mathrm{PPF}^{2} \times \mathrm{time}^{3}$ & $-1.5219 \times \mathrm{E}-08$ \\
20 & $\mathrm{CO}_{2} \times \mathrm{PPF}^{2} \times \mathrm{time}^{2}$ & $1.06285 \times \mathrm{E}-09$ \\
\hline
\end{tabular}

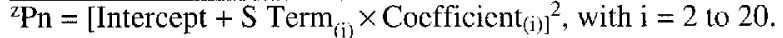

'Independent variables: time, 10 to $20 \mathrm{~d}$ after planting; $\mathrm{CO}_{2}$ level, 600 to $1500 \mu \mathrm{mol} \cdot \mathrm{mol}^{-1}$; and PPF, 600 to $1100 \mu \mathrm{mol} \cdot \mathrm{m}^{-2} \cdot \mathrm{s}^{-1}$.

normalized to a scale of 0 to 1 for use with a unipolar sigmoidal activation function (Nelson and Illingworth, 1991). Next, NN architecture was determined by grouping computing elements (neurons) into layers and connecting them. Neurons were connected such that the output of elements in one layer served as the input signal to elements in the next layer.

The training phase followed NN crcation. Our data set containing input conditions and the corresponding Pn response was used for supervised learning (Sarle, 1994). In the training phase, weights between the connected elements were adjusted iteratively until the NNs output matched the measured $P n$ for a given set of environmental conditions. This process continued until all the data had been incorporated into the training and an $R^{2}$ for each $\mathrm{NN}$ architecture was calculated. Next, we altered $N N$ architecture and repeated the training process. Parameters we manipulated included number of nodes in the hidden layer, number of connections among the nodes, and steepness of the activation function. A review of NN terminology and topology can be found in Eberhart and Dobbins (1990). Information describing the specific phases for NN development has also been reported (Bailey and Thompson, 1990b).

\section{Results}

Model specifics. Canopy Pn data collected from the first experiment were used to develop the statistical and NN models. In both approaches, $\mathrm{Pn}$ was determined as a function of three independent, input variables: 1) $\mathrm{CO}_{2}$ level, 2) PPF, and 3) time after planting. It was not possible to model Pn throughout the entire growth cycle (days 0 to 20) because Pn was not detectable before day 10 due to small plant size in their lag phase of development.

The function used to model canopy Pn took the form of a complex polynomial with 20 terms (Eq. [2]). Table 1 contains specific information regarding each term in the model. The regression model fit the experimental data set with an $R^{2}=0.994$.
Pn $=\left[\text { Intercept }+\sum \operatorname{Term}_{(i)} \times \text { Coefficient }_{(i)}\right]^{2}, i=2$ to 20

In general, first-, second--, and third-order terms containing time (days after planting) were significant in the statistical model. This result appears reasonable since a third-order polynomial may approximate a sigmoid-type response curve. Only first-order effects of $\mathrm{CO}_{2}$ level were significant, whereas first- and second-order terms of PPF were included in the model. The statistical model is valid only when $\mathrm{CO}_{2}, \mathrm{PPF}$, and time are all within ranges of the treatment combinations used to collect the original data set.

A NN model for canopy Pn was developed as an alternative to the traditional statistical approach. Final architecture included a simple, fully connected, three-layer $N N$, with three nodes in the input layer (to represent $\mathrm{CO}_{2}, \mathrm{PPF}$, and time), three nodes in the hidden layer, and one node in the output layer (to represent canopy $\mathrm{Pn}$ ). The resulting NN model had an $R^{2}$ of 0.96 and a maximum delta of 0.075 . Variations of this NN architectures were attempted, but model $R^{2}$ did not change significantly with other designs.

USING THE MODELS. Both models were used to predict the maximum and minimum $\mathrm{Pn}$ that could be achieved on any given day during a crop growth cycle (Fig. 1). It follows that any desired Pn between the daily maximum and minimum values also is attainable by setting $\mathrm{CO}_{2}$ and PPF at some appropriate levels. The maximum and minimum $P n$ predicted for each day depended on our experimental ranges of $\mathrm{CO}_{2}$ and PPF. For example, certainly $\mathrm{Pn}<0$ can be obtained if respiring plants are contained in total darkness. However, 0 PPF was out of the boundaries of the original data set used to generate the models.

Both models revealed a continuous sigmoidal-type relationship to describe canopy Pn over time (Fig. 1). However, the curvature of the NN response model was more gradual than that of the statistical model. The models predicted a maximum Pn potential of $\approx 0$ (on

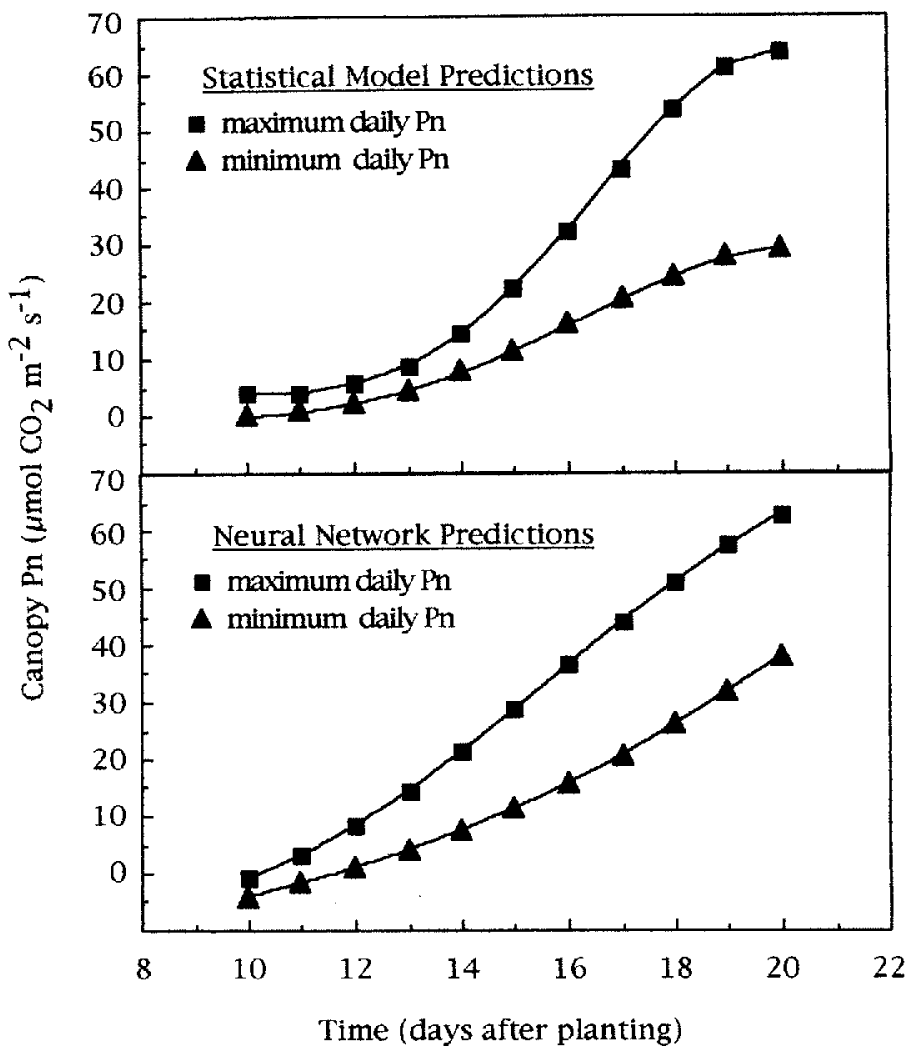

Fig. 1. Daily range of attainable canopy photosynthesis ( $\mathrm{Pn}$ ) rates as predicted by the statistical and ncural nctwork models and with dependent variables held within specific limits. 


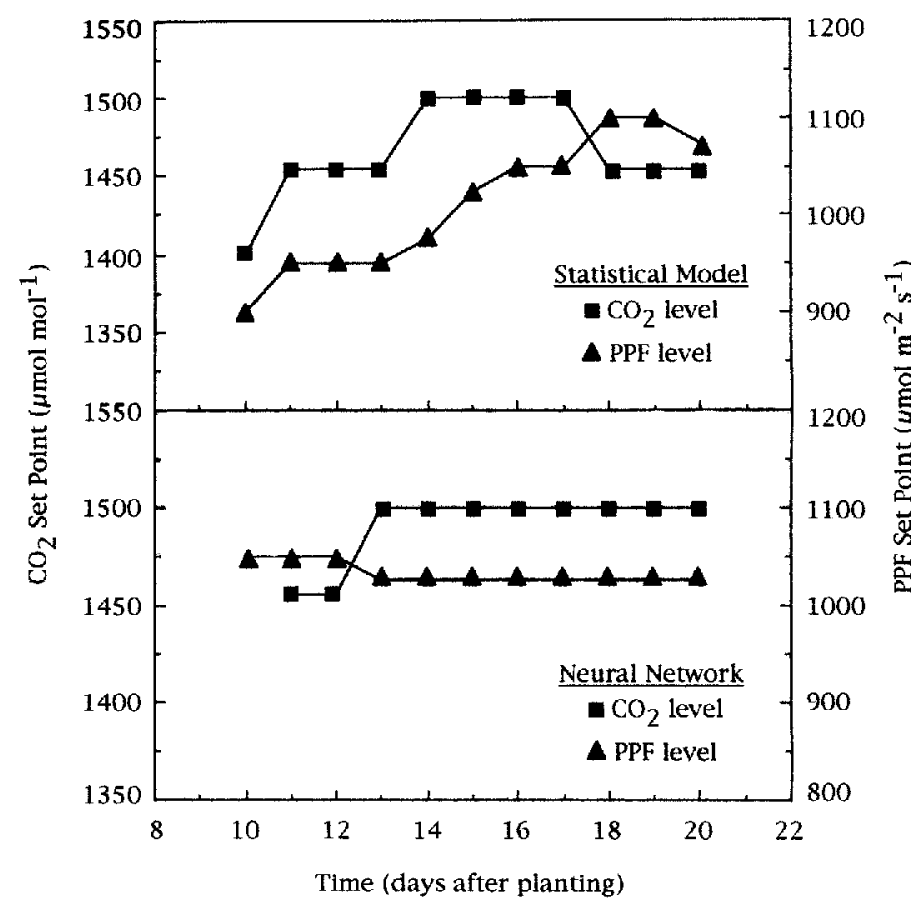

Fig. 2. Daily combinations of $\mathrm{CO}_{2}$ and PPF setpoints required to maintain maximum canopy photosynthesis (Pn), as predicted by the statistical and neural network models.

day 10 ) up to $65 \mu \mathrm{mol} \cdot \mathrm{m}^{-2} \cdot \mathrm{s}^{-1} \mathrm{CO}_{2}$ (on day 20 ). It should be noted that 0 Pn reflected the sensitivity of our environmental control equipment. Hence, the canopy was photosynthetically active, but this activity was not detectable until after day 10 .

In addition to predicting the daily range of possible $\mathrm{Pn}$, the models also were used to determine setpoints for $\mathrm{CO}_{2}$ and $\mathrm{PPF}$ required to achieve maximum potential $\mathrm{Pn}$ for each day in the growing cycle (Fig. 2). Daily maximum $P n$ values were available from the data used to generate Fig. 1 . Starting with the desired maximum Pn, models were solved iteratively to determine $\mathrm{CO}_{2}$ and PPF combinations required each day to obtain the predicted maximum $\mathrm{Pn}$ (Fig. 2). This day-to-day optimization approach is potentially valuable since energy and resource savings may be realized without compromising crop growth.

Suggested $\mathrm{CO}_{2}$ and PPF setpoints differed substantially between the statistical and NN models (Fig. 2). According to the statistical model, PPF should be set at $\approx 900 \mu \mathrm{mol} \cdot \mathrm{m}^{-2} \cdot \mathrm{s}^{-1}$ on day 10 , and then increased gradually to $1100 \mu \mathrm{mol} \cdot \mathrm{m}^{-2} \cdot \mathrm{s}^{-1}$ by day 18 . This demonstrates that a phasic environmental control strategy may be applicable for lettuce stands without compromising potential Pn. The statistical model suggested very slight alterations in daily $\mathrm{CO}_{2}$ setpoints, starting with $1400 \mu \mathrm{mol} \cdot \mathrm{mol}^{-1}$ on day 10 and increasing to 1500 $\mu \mathrm{mol} \cdot \mathrm{mol}^{-1}$ by day 14 (Fig. 2 ). It is unknown if such a subtle change in $\mathrm{CO}_{2}$ levels realistically affects crop development. The statistical model also revealed that $\mathrm{CO}_{2}$ andPPF could be decreased near the end of the growth cycle (day 19) without significant reduction of maximum Pn.

According to the $\mathrm{NN}$ model, daily setpoints for $\mathrm{CO}_{2}$ and PPF could not be altered greatly and still achieve the maximum predicted Pn (Fig. 2). For all practical purposes, the NN suggested a constant PPF of 1040 to $1050 \mu \mathrm{mol} \cdot \mathrm{m}^{-2} \cdot \mathrm{s}^{-1}$ throughout the cropping cycle. Similarly, a fairly constant $\mathrm{CO}_{2}$ setpoint of $1500 \mu \mathrm{mol} \cdot \mathrm{mol}^{-1}$ was determined from the NN model. The NN model also implied no Pn response to $\mathrm{CO}_{2}$ on day 10 . Therefore, there was no suggested $\mathrm{CO}_{2}$ setpoint for that day.

VAIIDATING THE MODELs. In a follow-up experiment, daily $\mathrm{CO}_{2}$ and PPF levels were set to those conditions determined by the statistical and NN models to maintain maximum Pn (Fig. 2). Canopy Pn was measured under these conditions and compared to the daily, predicted maximum target Pn (Fig. 1). Overall, the statistical model predicted canopy Pn more accurately than the NN model (Fig. 3 and Fig. 4). In the statistical approach, average percent difference between predicted $\mathrm{Pn}$ and actual $\mathrm{Pn}$ was $12.3 \%$, when results were averaged over an 11-d evaluation period (days 10 to 20 after planting). In comparison, average percent difference for the NN model averaged $24.6 \%$ over a 10 - $d$ period (days 11 to 20 ).

For both models, measured canopy Pn typically was less than the models had predicted (Fig. 3). Furthermore, divergence between predicted and actual Pn values became greater as the canopy aged (Fig. 4). For the statistical approach, actual Pn began to diverge from the predicted values by day 16 . The situation was more extreme in the NN model, with divergence beginning by day 12 .

\section{Discussion}

The Pn models reported in this study are relatively simple compared to those that ultimately will be required for a CELSS. Of the 10 or more proposed CELSS candidate species (Hoff et al., 1983; Salisbury and Clark, 1996), all will require models to predict total crop response (e.g., Pn, transpiration, respiration, and edible biomass yield and quality). These models must predict crop response as a function of multiple, environmental variables. For example, models must simultaneously incorporate variables such as $\mathrm{CO}_{2}$, PPF, temperature, spectral quality (for reproductive crops), photoperiod, and humidity. Rootzone variables such as nutritional regimes and $\mathrm{pH}$ also have been shown to be important when quantifying crop responses (Frick and Mitchell, 1993; Frick et al., 1994).

These dynamic models were developed in part to test the possibil-

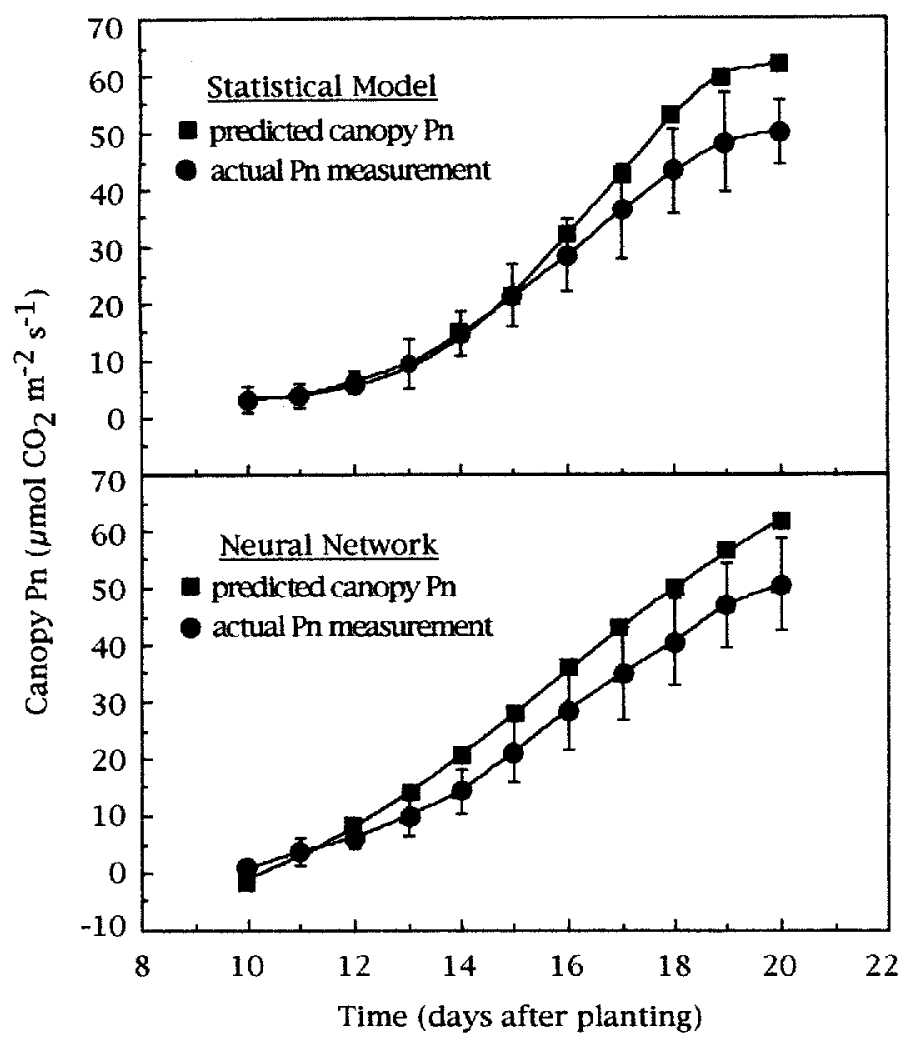

Fig. 3. Comparison between predicted maximum photosynthesis (Pn) values and actual Pn measurements for the statistical and neural network models. Vertical bars are standard errors of the mean. 


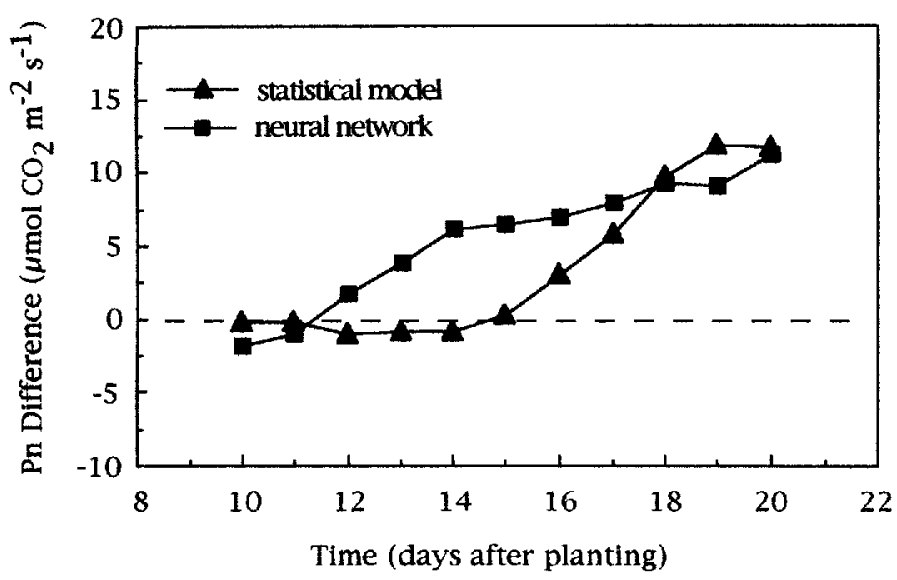

Fig. 4. Daily differences between predicted and actual values of canopy photosynthesis $(\mathrm{Pn})$ for the statistical and neural network models. Pn differences that approach 0 reflect greater accuracy in Pn prediction.

ity of using a phasic-control strategy for managing environmental variables during crop growth. In a phasic-control approach, environmental conditions may be modified incrementally throughout the entire growth cycle of the crop. For example, optimal conditions on day 5 might differ from those required on day 15 . For our purposes, the phasic time interval was daily. However, as the accuracy of the model increases, the time interval between environmental modifications could be decreased to hours, or perhaps minutes, depending on how quickly equilibrium can be achieved within the environment. The advantage of a phasic approach to controlled environment agriculture involves potential resource savings, especially with regard to electrical energy.

A logical next stepinvolves research toward a real-time, active-control scenario. Active control refers to a situation in which a desired level of $\mathrm{Pn}$ is predetermined, and a real-time feedback signal triggers automatic changes in environmental conditions until the target $P n$ is obtained. In an active-control scenario, the desiredPnis maintainedorchanged at will. An active-control algorithm for Pn could be useful in a CELSS whenever immediate modifications of air revitalization rates are required. Active control over canopy Pn not only requires real-time crop response models, but also computerized data acquisition and environmental control hardware that can react rapidly and accurately.

We chose to test a NN model as an alternative approach to modeling crop response data. We were interested to see whether a simple NN could describe a complex crop response such as canopy Pn. The purpose of a NN is to capture the relationship between system inputs and corresponding outputs. Once this relationship is encoded into a NN, it can be used to predict future values. It also can be used to control the inputs of a system to achieve a desired output. Previous agricultural and horticultural studies have successfully used NNs to determine apple quality (Bochereau et al., 1992) and to predict fieldgrown corn yields (Uhrig et al., 1992). NNs also have been used to model pine tree survival (Guan and Gertner, 1991) and to recognize chrysanthemum nodes for robotic micropropagation (Davis, 1991).

Although success with our simple NN to predict Pn was limited, there are several reasons why this modeling technique may hold potential for future CELSS applications. NNs are reportedly well suited to applications that contain the following characteristics: 1) measured response dependent on multiple, interacting parameters; 2) data set is incomplete or error prone (e.g., due to biological variability, calibration drift, etc.); and 3) the true biological function either is unknown or expensive to discover through experimental techniques (Bailey and Thompson, 1990a).

An interesting result of our study regards the increasing diver- gence between predicted and actual Pn values over time (as seen with both models, Figs. 3 and 4). One explanation for this divergence may involve chaotic-type effects, where subtle environmental differences early in the growth cycle may result in unpredictable, potentially large discrepancies in the expected crop response. In chaos theory this concept commonly is referred to as the butterfly effect (Gleick, 1987).

More research is needed before attributing our observed divergence entirely to chaos. However, assuming chaos plays even a minor role in crop responses, then CELSS development will encounter a new level of complexity. Not only will it be difficult to create consistent and accurate crop models, but long-term system stability also might be questionable. For instance, suppose an environmental perturbation occurs in a CEI.SS (e.g., setpoint changes, equipment malfunction, etc.), then the following questions arise: What magnitude and duration of disturbance causes a significant deviation from model predictions? During which crop growth phase (if any) is it safe for such a disturbance to occur? To answer these questions, models of all types should be explored and refined.

\section{Literature Cited}

Allen, D.H. and W.E. Haisler. 1985. Introduction to aerospace structural analysis. Wiley, New York.

Bailey, D. and D. Thompson. 1990a. How to develop neural-network applications. AI Expert (June):38-45

Bailey, D. and D. Thompson. 1990b. Developing neural-network applications. AI Expert (September):34 41

Bochereau, L., P. Bourgine, and B. Palagos. 1992. A method for prediction by combining data analysis and neural networks: Application to prediction of apple quality using near infra-red spectra. J. Agr. Eng. Res. 51:207-216.

Box, G.E., W.G. Hunter, and J.S. Hunter. 1978. Statistics for experimenters: An introduction to design, data analysis, and model building. Wiley, New York.

Davis, P.F. 1991. Orientation-independent recognition of chrysanthemum nodes by an artificial neural network. Computers Electronics Agr. 5:305-314.

Eberhart, R.C. and RW. Dobins. 1990. Early neural network development history: The age of camelot. Engineering in medicine and biology magazine: The quarterly magazine of the engincering in medicine and biology society. $9(3)$.

Frick, J. and C.A. Mitchell. 1993. Stabilization of $\mathrm{pH}$ in solid-matrix hydroponic systems. HortScience 28(10):981-984

Frick, J., S. Nielsen, and C.A. Mitchell. 1994. Yield and seed oil content response of dwarf, rapid cycling brassica to nitrogen treatments, planting density, and $\mathrm{CO}$, enrichment. J. Amer. Soc Hort. Sci. 119(6):1137-1143.

Guan, B.T. and G. Gertner. 1991. Modeling red pine tree survival with an artificial neural network. For. Sci. 37(5):1429-1440.

Gleick, J. 1987. Chaos: Making a new science. Penguin Books, New York.

Hoagland, D.R. and D.I. Arnon. 1950. The water culture method for growing plants without soil Univ. Calif., Berkeley, Agr. Expt. Sta. Circ. 347.

Hoff, J.E., J.M. Howe, and C.A. Mitchell. 1983. Nutritional and cultural aspects of plant species selection for a regenerative life support system. NASA Contractor Rpt. 166324.

Holman, J.P. 1984. Experimental methods for enginecrs. 4th cd. McGraw-Hill, New York

Knight, S.L., C.P. Akers, S.W. Akers, and C.A. Mitchell. 1988. Minitron II system for precise control of the plant growth environment. Photosynthetica 22:90 98.

Machado, R.Z., M.F. Tenorio, F. Manael, and R.M. Jose. 1992. Neural network error corrector for binary messages on hydroacoustic channels. J. Oceanic Eng. 17(4):369-375.

McCulloch, W.S. and W.H. Pitts. 1943. A logical calculus of the ideas immanent in nervous activity. Bul. Math. Biophys. 5:115-133.

Milthorpe, F.L. and J. Moorby. 1979. An introduction to crop physiology. Cambridge Univ. Press.

Nelson, M.C. and W.T. Illingworth. 1991. A practical guide To neural nets. Addison-Wesley, Reading, Mass.

Ripley, B.D. 1993. Statistical aspects of neural networks in networks and chaos: Statistical and probabilistic aspects. Chapman and Hall Press, London.

Salisbury, F.B. and M.A. Clark. 1996. Choosing plants to be grown in a controlled environment life-support system based upon attractive vegetarian diets. Life Support Biosphere Sci. 2:169179.

Sarle, W.S. 1994. Neural networks and statistical models. Proc. 19th Annu. SAS Uscrs Group Intl. Conf. SAS Inst., Cary, N.C

Steel, G.D. and J.H. Torrie. 1980. Principles and procedures of statistics: A biometric approach. 2nd ed. McGraw-Hill, New York.

Uhrig. J.W., B.A. Engel, and W.L Baker. 1992. An application of neural networks: Predicting corn yields. Computers in agricultural extension programs. Proc. 4th Intl. Conf. Amer. Soc. Agr. Eng., Orlando, Fla.

Weiss, S.M. and C.A. Kulikowski. 1991. Computer systems that learn. Morgan Kaufman, San Mateo, Calif.

White, H. 1992. Artificial neural networks: Approximation and learning theory. Blackwell Press, Oxford, U.K. 